\title{
The Root Cause of the Overheating Problem
}

\author{
Meng-Sing Liou* \\ NASA Glenn Research Center, Cleveland, $\mathrm{OH} 44135$, USA
}

\begin{abstract}
Previously we identified the receding flow, where two fluid streams recede from each other, as an open numerical problem, ${ }^{1}$ because all well-known numerical fluxes give an anomalous temperature rise, thus called the overheating problem. This phenomenon, although presented in several textbooks, ${ }^{2,3}$ and many previous publications, has scarcely been satisfactorily addressed and the root cause of the overheating problem not well understood. We found that this temperature rise was solely connected to entropy rise and proposed to use the method of characteristics to eradicate the problem. ${ }^{4,5}$ However, the root cause of the entropy production was still unclear. In the present study, we identify the cause of this problem: the entropy rise is rooted in the pressure flux in a finite volume formulation and is implanted at the first time step. It is found theoretically inevitable for all existing numerical flux schemes used in the finite volume setting, as confirmed by numerical tests. This difficulty cannot be eliminated by manipulating time step, grid size, spatial accuracy, etc, although the rate of overheating depends on the the flux scheme used. Finally, we incorporate the entropy transport equation, in place of the energy equation, to ensure preservation of entropy, thus correcting this temperature anomaly. Its applicability is demonstrated for some relevant $1 \mathrm{D}$ and $2 \mathrm{D}$ problems. Thus, the present study validates that the entropy generated $a b$ initio is the genesis of the overheating problem.
\end{abstract}

\section{Introduction}

$\mathrm{T}$ HE overheating problem is one of the open CFD problems that yet to be understood and correctly computed; it has been found in two scenarios occurring in two opposite situations-one related to colliding compressive flows and the other to receding expansive flows. The former results either from reflection of a flow from a wall or equivalently from two colliding flows, manifested as a spike in temperature, and is first studied extensively by Noh. ${ }^{6}$ This overheating problem still challenges CFD researchers today, see for example the books by Toro ${ }^{2}$ and LeVeque. ${ }^{3}$ The receding flow problem, while less known, occurs during rarefaction: for example, two streams recede from each other, thereby creating a rarefying region between them, where all thermodynamic properties-pressure, density, and temperature should be decreasing. Unexpectedly, a numerical anomaly arises for an isentropic expansion process during which the calculated temperature not only departs significantly from the correct profile, but a noticeably odd increase appears in the center region, as depicted in Fig. 1. Two typical solutions are displayed-between the diffusive HLLE method and the sharp Godunov method, ${ }^{2}$ both showing the same oddity in temperature, although one is more smeared than the other. Toro ${ }^{2}$ states "... both pressure and density are close to zero and thus small errors will be exaggerated by their ratio" (p. 227). However, we showed in our previous studies ${ }^{1,4,5}$ that this anomaly occurs even in early times when both pressure and density are still $\mathrm{O}(1)$.

Based on the previous study, ${ }^{5}$ the temperature rise is established to have a strong connection to the entropy rise. Hence, an alternative path is to study the entropy evolution for gaining insight into the problem. This motivates us to pursue the present study with the objective to analytically uncover the root cause why the entropy is generated by numerical fluxes in an isentropic flow. Then, an entropy-preserving system, which replaces the energy equation with the entropy equation, is implemented to confirm that the temperature anomaly is indeed eradicated.

The paper is organized as follows. We first give a brief review of our previous attempts and findings, then we develop an analytical framework to look into the entropy change. Finally we incorporate the entropy equation in the solution procedure and confirm that if the entropy is preserved then the temperature anomaly disappears.

\footnotetext{
${ }^{*}$ Senior Technologist, Aeropropulsion Division, MS 5-11, 21000 Brookpark Road, Cleveland, OH 44135. Fellow.
} 


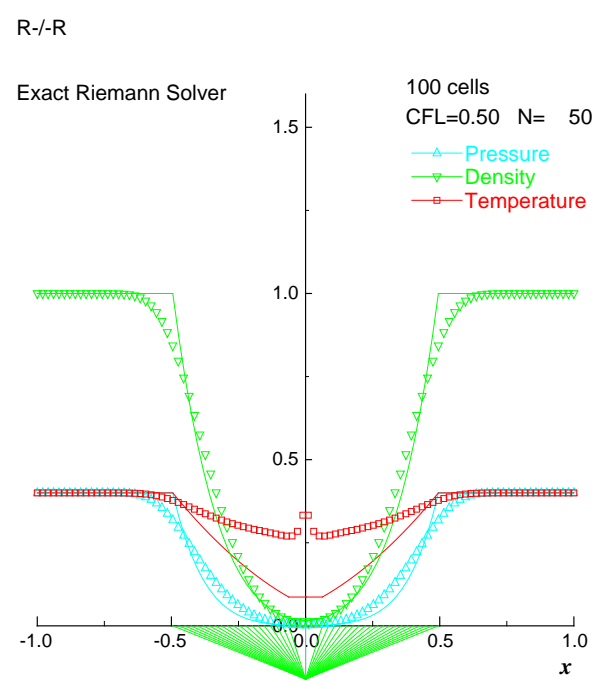

(a) Godunov.
$\mathrm{R}-\mathrm{-}-\mathrm{R}$

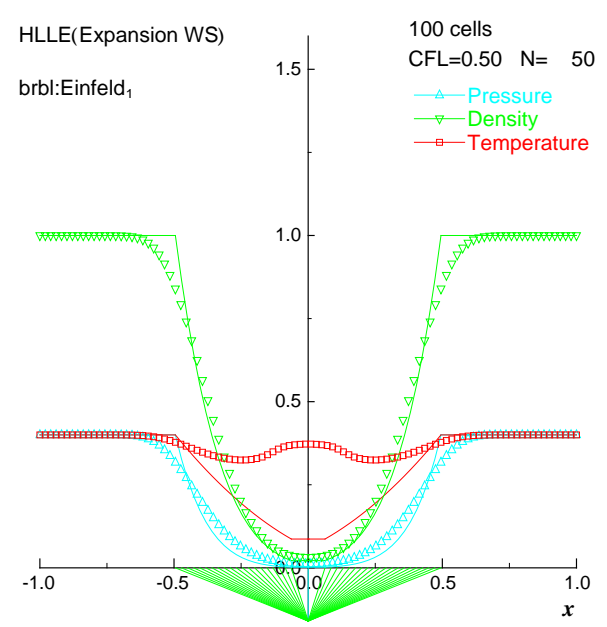

(b) HLLE.

Figure 1. Receding flows computed on a grid of 100 cells, with the initial condition, $(\rho, p, u)_{L}=(1.0,0.4,-2.0)$ and $(\rho, p, u)_{R}=(1.0,0.4,2.0)$ Ref. $\left[{ }^{5}\right]$.

\section{Statement of the Problem}

\section{II.A. Definition of the overheating problem}

For the overheating problem discussed in this paper, we shall focus on the receding problem, specifically a Riemann problem with the following initial condition:

$$
\begin{aligned}
& u_{L}<0, \rho_{L}, p_{L} \\
& u_{R}>0, \rho_{R}, p_{R}
\end{aligned}
$$

where the "L" and "R" states may be of the same or different magnitudes. We shall confine our discussion to the case of equal magnitudes, $u_{L}=-u_{R}, \rho_{L}=\rho_{R}, p_{L}=p_{R}$. The strength of rarefaction is defined by the relatively Mach number $\left|M_{R}-M_{L}\right|$.

Our interest in studying this seemly simple problem is motivated by the desire to understand the numerical mechanism responsible for the overheating. A brief summary of our previous efforts in this regard will provide a useful guide for gaining insight into the problem.

\section{II.B. Previous findings $\mathbf{s}^{1,4,5}$}

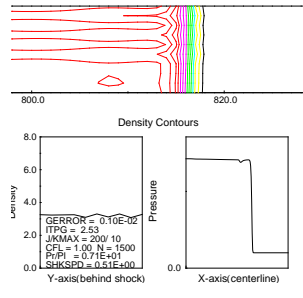

(a) Godunov, $\mathrm{CFL}=1$.



(b) Roe-E, CFL=1.

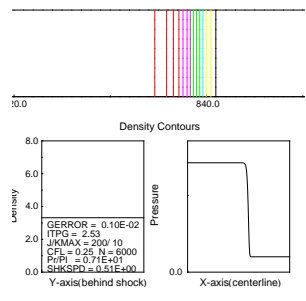

(c) Godunov, $\mathrm{CFL}=1 / 4$

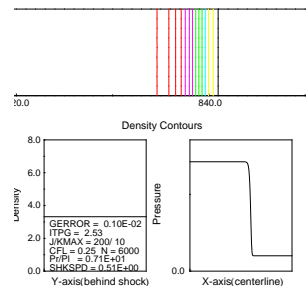

(d) Roe-E, CFL=1/4.

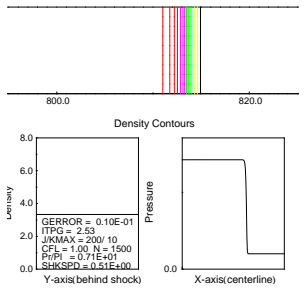

(e) $\mathrm{AUSM}^{+}$-up, $\mathrm{CFL}=1$, grid perturbed by $10 \%$.

Figure 2. A plane shock wave traveling in a constant-area channel in which the center grid line is perturbed at alternating even and odd points with a magnitude of $1 \%$ of grid spacing $\Delta \mathrm{Y}$, results are obtained using the Godunov, Roe, and $\mathrm{AUSM}^{+}$-up with various CFL numbers. The AUSM ${ }^{+}$-up solution remains clean even with a $10 \%$ grid perturbation (Ref. $\left.\left[{ }^{1}\right]\right)$.

In the previous study of some "difficult" numerical problems, we characterize the difficulties into two categories: (1) fundamental and (2) operational. The "operational" category refers to those problems remediable by change of operational procedures such as methods, mesh distribution, time step, limiters, etc. The carbuncle problem, which has remained a controversial topic, is an example of such operational problem, based on the evidence in our study: sample results shown in Fig.2 demonstrate that the shock anomalies associated with different methods can be made to 
disappear by simply changing the time step. The difficulty in the "fundamental" category, on the other hand, cannot be overcome by simply changing numerical parameters and/or switching numerical schemes. The receding problem considered in this paper belongs to this category. Figure 3 shows the typical calculated temperature profiles for subsonic and supersonic flows, in which again the temperature rises to a peak value at the center between two streams. From our previous studies, this phenomenon is universal irrespective of flux functions used, although quantitative differences exist among them.

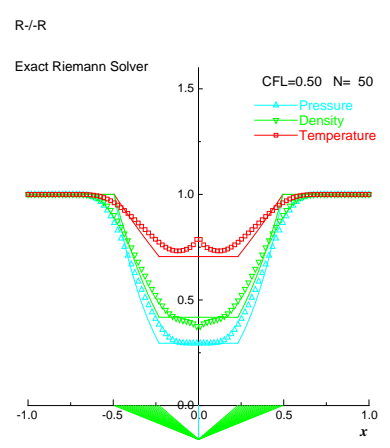

(a) $\mathrm{M}=0.8,100$ cells.

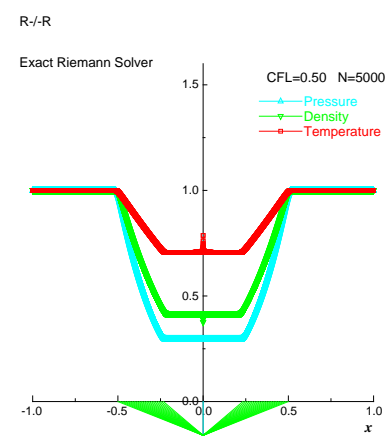

(b) $\mathrm{M}=0.8,10,000$ cells.

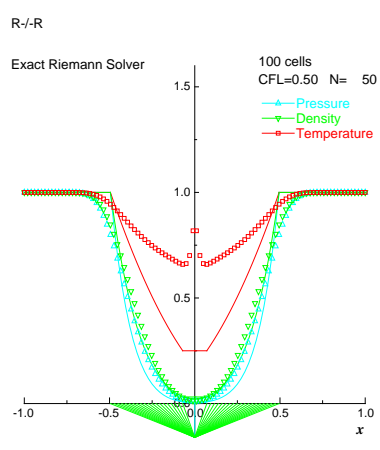

(c) $\mathrm{M}=2.5,100$ cells.

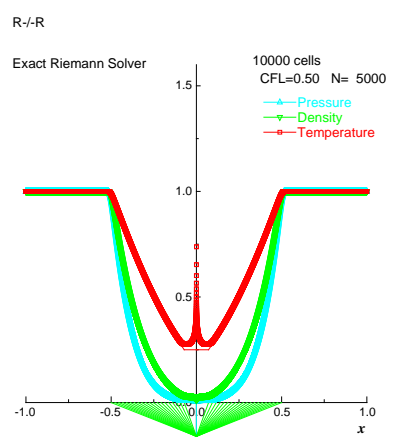

(d) $\mathrm{M}=2.5,10,000$ cells.

Figure 3. Solutions of receding flows moving at $M=0.8$ and 2.5 by the Godunov method on 100 and 10,000 cells respectively (Ref. $\left.\left[{ }^{1}\right]\right)$.
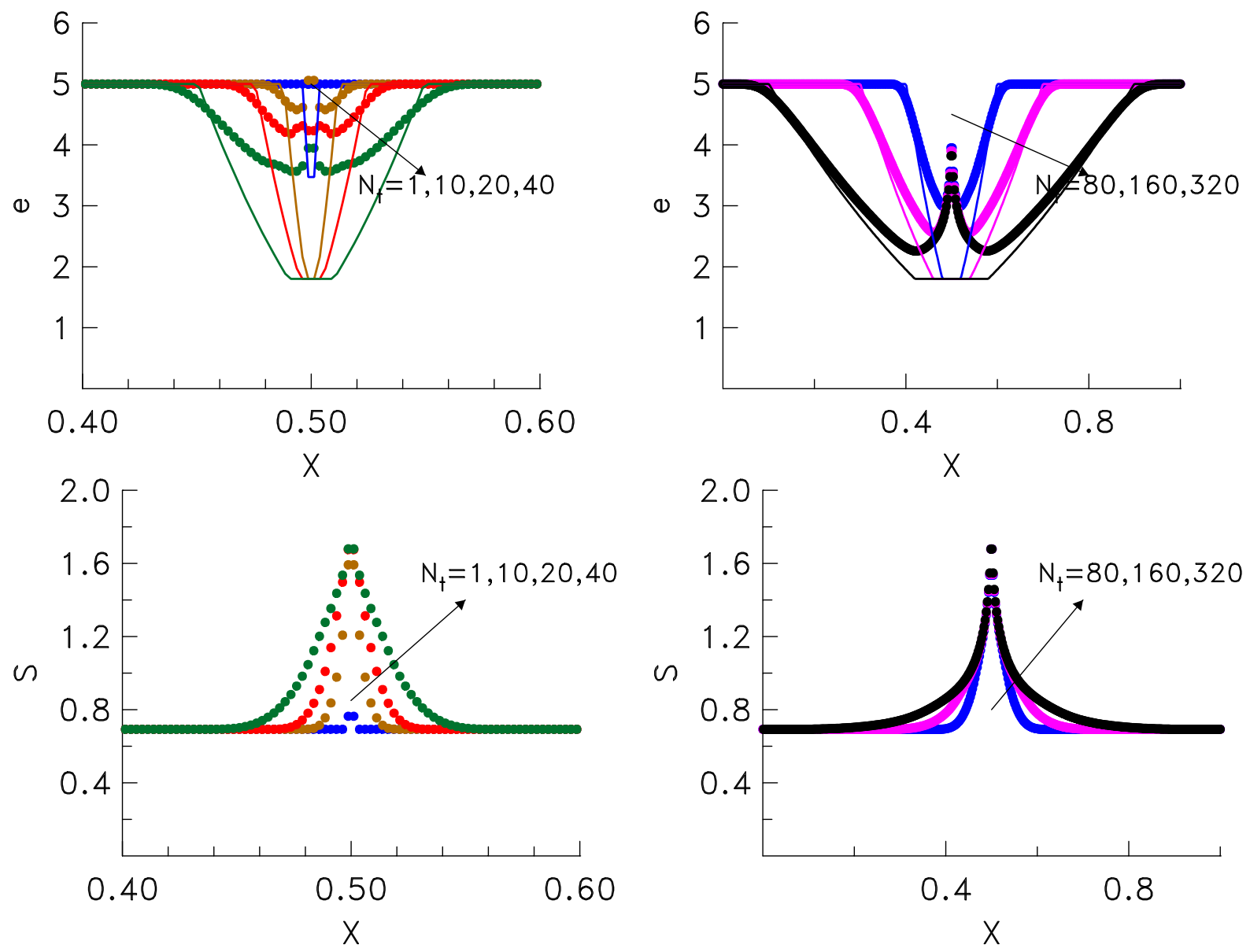

Figure 4. Evolution of temperature and entropy at various time slices (Ref. $\left.\left[{ }^{5}\right]\right)$. Solid lines for the e vs. $x$ plots are the exact solutions. 
The incorrect temperature behavior is found to be related to entropy production, ${ }^{4}$ as depicted in Fig. 4 . We see that (1) the entropy is increased at the first moment at the center, clearly violating the second law of thermodynamics for this smooth flow, and (2) this entropy violation continues to spread spatially. Furthermore, the entropy peak at the center reaches a maximum value and then stays at a saturated value, which is found to be rather insensitive to the number of mesh points used. This explains why the overheating is not shrunk by changing the order of accuracy or mesh size. It also strongly suggests that in order to resolve this unphysical overheating problem in the receding flow, preservation of the entropy is the key. This viewpoint is further confirmed that if the entropy condition is enforced, then the temperature indeed behaves correctly, as will be shown later.

The link of the temperature increase to the entropy production is further analyzed subsequently, ${ }^{5}$ in which it is argued that the overheating is inevitable in the current finite volume discretization framework. This link of entropy production and temperature rise is found to follow a numerical heat diffusion model,

$$
T d s=\kappa d T
$$

Or

$$
\Delta s=s(x+\Delta x)-s(x)=\kappa \log \frac{T(x+\Delta x)}{T(x)}
$$

Figure 5 shows the variation of these two calculated quantities between two neighboring points for the entire computation domain, confirming that the relationship in Eq. 4 holds, the entropy increases with temperature, and the slope manifested as the numerical diffusivity of this calculation is nearly a constant. The points further away from the center correspond to the region of large changes in entropy and temperature, i.e., those near the center of the receding flow.



Figure 5. Temperature variation $\Delta_{x} \log T$ vs. entropy variation $\Delta_{x} s$.

To remove this temperature anomaly in the receding flow, the method of characteristics (MOC) was used to replace the conventional discretized system to advance the solution; this approach has proven to be successful in correcting the temperature overheating. 5

However, the reason why the entropy is produced in the first place remains a mystery. This motivates the current study, as will be described in what follows.

\section{Formulation}

As evident from the previous study, the entropy is generated and plays a unique role in the overheating problem. The key is to find how the entropy is produced in the discretized conservation laws employed for the numerical solution. A fundamental thermodynamic equation for an ideal gas gives

$$
T d s=d e-\frac{p}{\rho^{2}} d \rho=e\left(\frac{d p}{p}-\gamma \frac{d \rho}{\rho}\right)=\frac{C_{v} T}{p}\left(d p-a^{2} \frac{d \rho}{\rho}\right)=C_{v} T d\left(\log \frac{p}{\rho^{\gamma}}\right)
$$

Or

$$
d s=C_{v} d\left(\log \frac{p}{\rho^{\gamma}}\right)=\frac{C_{v}}{p}\left(d p-a^{2} d \rho\right)
$$

$$
4 \text { of } 10
$$


The first equality in Eq. 6 allows us to define the usual entropy variable for an ideal gas as, (omitting an integration constant without loss of generality)

$$
s=C_{v} \log \frac{p}{\rho^{\gamma}}, \quad C_{v}=\frac{R}{\gamma-1}
$$

where $R$ is the gas constant. The second equality in Eq. 6 allows to relate the time rate of change of entropy to that of the conservation laws via pressure and density. From the energy equation, we find

$$
\frac{\partial \rho E}{\partial t}=\frac{\partial}{\partial t}\left(\rho e+\frac{(\rho u)^{2}}{2 \rho}\right)=\frac{1}{\gamma-1}\left(\frac{\partial p}{\partial t}-a^{2} \frac{\partial \rho}{\partial t}\right)+\left(\frac{a^{2}}{\gamma-1}-\frac{u^{2}}{2}\right) \frac{\partial \rho}{\partial t}+u \frac{\partial \rho u}{\partial t}
$$

Combining Eqs. 6 and 8 yields

$$
\frac{p}{R} \frac{\partial s}{\partial t}=\frac{\partial \rho E}{\partial t}-\left(\frac{a^{2}}{\gamma-1}-\frac{u^{2}}{2}\right) \frac{\partial \rho}{\partial t}-u \frac{\partial \rho u}{\partial t}
$$

Clearly this equation characterizes the balance of entropy change and the changes of mass, momentum, and total energy. This is a very illuminating equation, from which we see how the entropy can be produced. Let us, for the purpose of illustration, consider the 1D conservation laws. The conclusion reached herein however holds for a multidimensional system, as will be shown later with some numerical results.

$$
\begin{array}{r}
\frac{\partial \rho}{\partial t}=-\frac{\partial \rho u}{\partial x} \\
\frac{\partial \rho u}{\partial t}=-\frac{\partial}{\partial x}\left(\rho u^{2}+p\right) \\
\frac{\partial \rho E}{\partial t}=-\frac{\partial \rho u H}{\partial x}
\end{array}
$$

Now substituting the above spatial derivatives in the balance equation Eq. 9, the continuous system becomes, after a simple algebraic manipulation,

$$
\frac{p}{R} \frac{\partial s}{\partial t}=-\frac{p}{R} u \frac{\partial s}{\partial x}
$$

Since $p / R \neq 0$, it simplifies to

$$
\frac{\partial s}{\partial t}+u \frac{\partial s}{\partial x}=0
$$

This is the familiar entropy equation, leading to the well-known result for a smooth flow-The entropy remains unchanged along the particle path.

The receding flow considered above is an isentropic flow initially and it should theoretically remain isentropic throughout according to Eq. 14. However all the numerical solutions predicted otherwise. The key difference between the theoretical and numerical results lies in the fact that the discrete version of Eqs. 10-12 is not consistent with the continuous system, as will become evident below.

Let us consider the finite volume discretization of Eq. 9, in which the right hand side is replaced by numerical fluxes at the cell faces, see the following schematic.

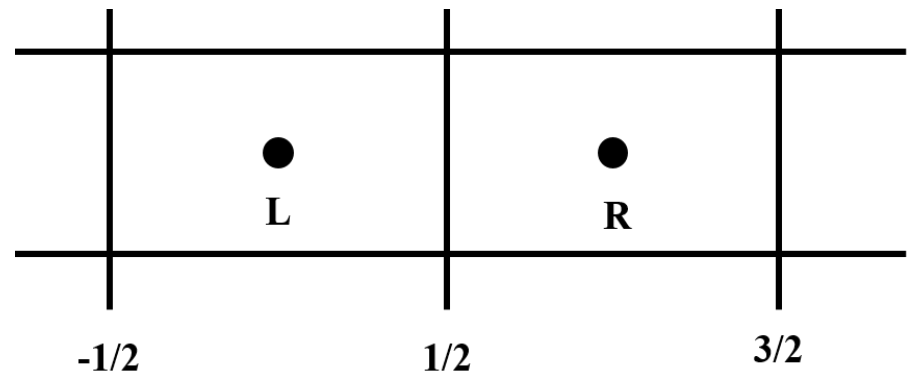

Figure 6. Schematic of finite-volume cells and indices. 
For the "R" cell bounded by faces " $3 / 2$ " and " $1 / 2$ ", with initial conditions: $u_{R}=-u_{L}=u_{0}>0, \rho_{R}=\rho_{L}=$ $\rho_{0}, p_{R}=p_{L}=p_{0}$, we obtain

$$
\oint_{\text {"R" }} \frac{p}{R} \frac{\partial s}{\partial t} d V=-\left[(\rho u H)_{3 / 2}-(\rho u H)_{1 / 2}\right]+\left(\frac{a_{0}^{2}}{\gamma-1}-\frac{u_{0}^{2}}{2}\right)\left[(\rho u)_{3 / 2}-(\rho u)_{1 / 2}\right]+u_{0}\left[\left(\rho u^{2}+p\right)_{3 / 2}-\left(\rho u^{2}+p\right)_{1 / 2}\right]
$$

where the coefficients associated with the momentum and mass flux differences are evaluated at the initial time. The fluxes for the face " $3 / 2$ " is simple, based on the consistency requirement $\left(F\left(U_{R}, U_{R}\right)=F\left(U_{R}\right)\right)$, we have

$$
\begin{aligned}
(\rho u H)_{3 / 2} & =\rho_{0} u_{0} H_{0}=\rho_{0} u_{0}\left(\frac{a_{0}^{2}}{\gamma-1}+\frac{u_{0}^{2}}{2}\right) \\
(\rho u)_{3 / 2} & =\rho_{0} u_{0} \\
\left(\rho u^{2}+p\right)_{3 / 2} & =\rho_{0} u_{0}^{2}+p_{0}
\end{aligned}
$$

And for the face " $1 / 2$ " where we have $u_{1 / 2}=0$, by virtue of anti-symmetry, the fluxes are

$$
\begin{aligned}
(\rho u H)_{1 / 2} & =0 \\
(\rho u)_{1 / 2} & =0 \\
\left(\rho u^{2}+p\right)_{1 / 2} & =m_{1 / 2}+p_{1 / 2}
\end{aligned}
$$

where the momentum flux $m_{1 / 2}$ and pressure flux $p_{1 / 2}$ can take different forms for different flux schemes; they are given in Table 1. For all the numerical fluxes tested, we find

$$
p_{1 / 2}=\epsilon p_{0}, \quad 1 \geq \epsilon \geq 0
$$

Note that the $\epsilon$ for the exact Riemann solver is valid up to the vacuum condition $\left(M_{0}=2 /(\gamma-1)\right.$ in this case). The values of $\epsilon$ of some flux schemes for $M_{0}=0.8$ are given in Table 1 for reference. By substitution of Eqs. 16 and eq:1/2, Eq. 15 reduces to

$$
\oint_{\text {" } R^{\prime \prime}} \frac{p}{R} \frac{\partial s}{\partial t} d V=u_{0}\left[\left(p_{0}-p_{1 / 2}\right)-m_{1 / 2}\right]=u_{0}\left[(1-\epsilon) p_{0}-m_{1 / 2}\right]
$$

The first term is non-negative, thus contributing an increase in entropy (since $p / R>0$ ), while the second term may lead to a decrease in entropy if $m_{1 / 2}>0$. This latter situation happens only when $u_{0}>\tilde{a}_{0}$ for the Roe scheme, but this results in violation of the entropy condition, which in turn leads to a negative temperature and failure of computation. Hence, this entropy (thus temperature) positivity condition requires that $m_{1 / 2} \leq 0$. Notice that the van Leer flux gives $m_{1 / 2} \leq 0$, while all other schemes, as shown in Table 1 , give $m_{1 / 2}=0, \forall M_{0}$. Hence, for an entropy-satisfying $(\Delta s \geq 0)$ flux scheme (implying $m_{1 / 2} \leq 0$ ), the entropy is generated within the first time step in cell "R". Similarly we get the identical result for the "L" cell bounded by faces "1/2" and "-1/2",

$$
\oint_{\text {'L } L^{\prime \prime}} \frac{p}{R} \frac{\partial s}{\partial t} d V=u_{0}\left[(1-\epsilon) p_{0}-m_{1 / 2}\right]
$$

with

$$
\begin{aligned}
(\rho u H)_{-1 / 2} & =-\rho_{0} u_{0}\left(\frac{a_{0}^{2}}{\gamma-1}+\frac{u_{0}^{2}}{2}\right) \\
(\rho u)_{-1 / 2} & =-\rho_{0} u_{0} \\
\left(\rho u^{2}+p\right)_{-1 / 2} & =\rho_{0} u_{0}^{2}+p_{0}
\end{aligned}
$$

This shows that the entropy increase occurs in both the "L" and "R" cells, symmetrically with respect to the face "1/2" straddling them. Based on the analysis developed above, it is the numerical flux, specifically the pressure flux, at the cell face that creates the entropy. The numerical pressure flux at the interface " $1 / 2$ " results in an imbalance of pressures on the opposite faces, thus creating a net increase in entropy. This imbalance in pressure will continue to develop in the neighboring cells at the following time steps so long as the flow is evolving (changing) spatially. Hence, the entropy generation and temperature rise continue to spread spatialwise. 
Table 1. Expression of $m_{1 / 2}$ and $p_{1 / 2}$ (or $\epsilon$ ) of $\epsilon$ in Eq. 18 for various numerical fluxes, which can be found in Ref. $\left[{ }^{2}\right]$.

\begin{tabular}{|c|c|c|c|c|c|} 
Flux Scheme & Exact Riemann & AUSM-family & Roe & Van Leer & Steger-Warming \\
\hline$m_{1 / 2}$ & 0 & 0 & $\rho_{0} u_{0}\left(u_{0}-\tilde{a}_{0}\right)$ & $m_{1 / 2, V L}$ & 0 \\
\hline$\epsilon, p_{1 / 2}=\epsilon p_{0}$ & $\left(1-\frac{\gamma-1}{2} M_{0}\right)^{\frac{2 \gamma}{\gamma-1}}$ & $\epsilon_{1 / 2, A U S M^{+}}$ & 1 & $\epsilon_{1 / 2, V L}$ & $\epsilon_{1 / 2, S-W}$ \\
\hline$\epsilon\left(M_{0}=0.8\right)$ & 0.295 & 0.071 & 1 & 0.056 & 0.040 \\
\hline
\end{tabular}

$$
\begin{gathered}
m_{1 / 2, V L}= \begin{cases}-\frac{1}{2} \rho_{0} u_{0} a_{0}\left(M_{0}-1\right)^{2}, & \text { if } M_{0}<1, \\
0, & \text { otherwise. }\end{cases} \\
\epsilon_{1 / 2, V L}= \begin{cases}\frac{1}{2}\left(M_{0}-1\right)^{2}\left(2+M_{0}\right), & \text { if } M_{0}<1, \\
0, & \text { otherwise. }\end{cases} \\
\epsilon_{1 / 2, A U S M^{+}}= \begin{cases}\frac{1}{2}\left(M_{0}-1\right)^{2}\left(2+M_{0}\right)-\frac{3}{8} M_{0}\left(M_{0}^{2}-1\right)^{2}, & \text { if } M_{0}<1, \\
0, & \text { otherwise. }\end{cases} \\
\epsilon_{1 / 2, S-W}= \begin{cases}\left(M_{0}-1\right)^{2}, & \text { if } M_{0}<1, \\
0, & \text { otherwise. }\end{cases}
\end{gathered}
$$

From the above result, we now conclude the following.

Theorem: Under a finite volume discretization, the entropy production for a receding flow has its origin in the pressure flux, established $a b$ initio within the first time step. This is true regardless of cell size, flux function scheme, or time step; however the amount of entropy produced will depend on the flux functions used and the receding velocity.

Remark 1: For an entropy-satisfying condition, i.e., $\partial s / \partial t \geq 0$, the momentum flux $m_{1 / 2}$ must be non-positive; otherwise a negative temperature will result, rendering the calculation terminated. This happens to the Roe fluxe in supersonic receding flow.

Remark 2: The above analysis can serve as a simple tool to examine whether a flux scheme satisfies the entropy or positivity condition.

Although the above analysis only proves that the entropy is generated within the first time step, our calculations confirm that the trend continues in time and extends spatially. In fact, we find from the calculations in $\left[{ }^{1}\right]$ that for $t>0$ : (1) the overheating error remains even after increasing the number of cells by 100 times and (2) the error increases with the magnitude of the separation velocity (the strength of rarefaction), also evident in Fig. 3.

We re-iterate that the continuous system does guarantee preservation of entropy, it is only in the spatially discretized system that the entropy is produced. With the root cause established, the remaining question as to how to eradicate this numerical anomaly becomes clear.

Clearly, in order to eliminate this entropy production and the overheating anomaly the usual finite volume framework has to be revisited and modified. An alternative is to use an approach that obeys the entropy equation. The method of characteristics (MOC) is one that inherently includes the entropy condition. The MOC was shown to be successful in eradicating the overheating problem in some relevant flow problems. ${ }^{5}$ However, the MOC is rather cumbersome in dealing with geometry and boundary conditions; moreover it is hard to extend to higher order (temporal and spatial ) accuracy.

If all is possible, we still prefer the conventional finite-volume formulation, the only requirement is to incorporate the entropy condition. This can be achieved by using the entropy equation to replace the energy equation.

\section{III.A. Use of the entropy equation}

The nonconservative entropy equation ${ }^{\mathrm{a}}$, Eq. 14, can be used along with the continuity and momentum equations in conservation form, and a simple upwind discretization is used.

$$
s_{i}^{n+1}=s_{i}^{n}-\frac{\Delta t}{\Delta x}\left[u_{i}^{+}\left(s_{i}^{n}-s_{i-1}^{n}\right)+u_{i}^{-}\left(s_{i+1}^{n}-s_{i}^{n}\right)\right], \quad u_{i}^{ \pm}=\left(u_{i}^{n} \pm\left|u_{i}^{n}\right|\right) / 2
$$

${ }^{\text {a }}$ Since an isentropic flow implies a smooth flow, conservation form is not necessary. 
Or a conservative form can be used by combining with the continuity equation, given as: ${ }^{b}$

$$
\frac{\partial \rho s}{\partial t}+\frac{\partial \rho u s}{\partial x}=0
$$

It now takes the place of the energy equation; it is simple and cast in the same framework as the other conservation equations. For example, the entropy flux ( $\rho u s)$ at the cell interface is evaluated exactly the same way as the other fluxes for the AUSM-family schemes. Figure 7 demonstrates that the result of enforcing the entropy equation for the $1 \mathrm{D}$ receding flow-maintaining constant entropy and eliminating the temperature rise; similar results are also obtained for other flux schemes. We also note that the conservative form of the entropy equation gives results indistinguishable from that of the nonconservative form.

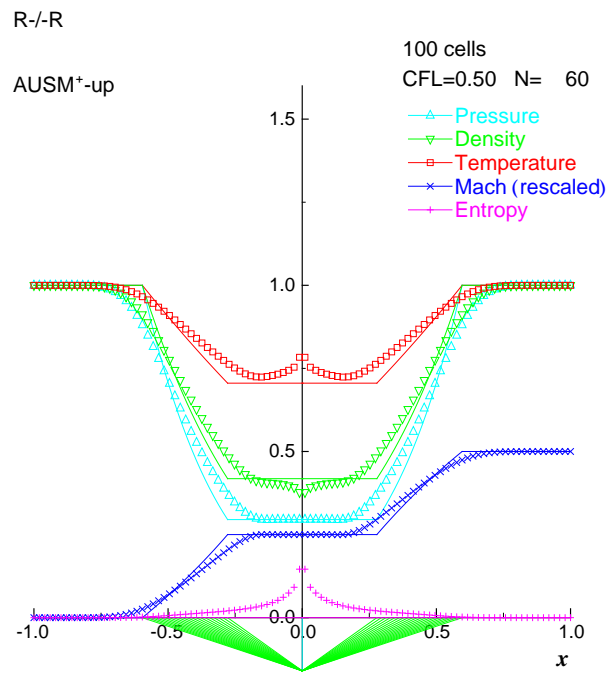

(a) $\mathrm{AUSM}^{+}$-up



(b) $\mathrm{AUSM}^{+}$-up with s-equation

Figure 7. Effects of enforcing the entropy equation. (a) $\mathrm{AUSM}^{+}$-up and (b) $\mathrm{AUSM}^{+}$-up with the entropy equation for the $1 \mathrm{D}$ receding flow problem (M=0.8).

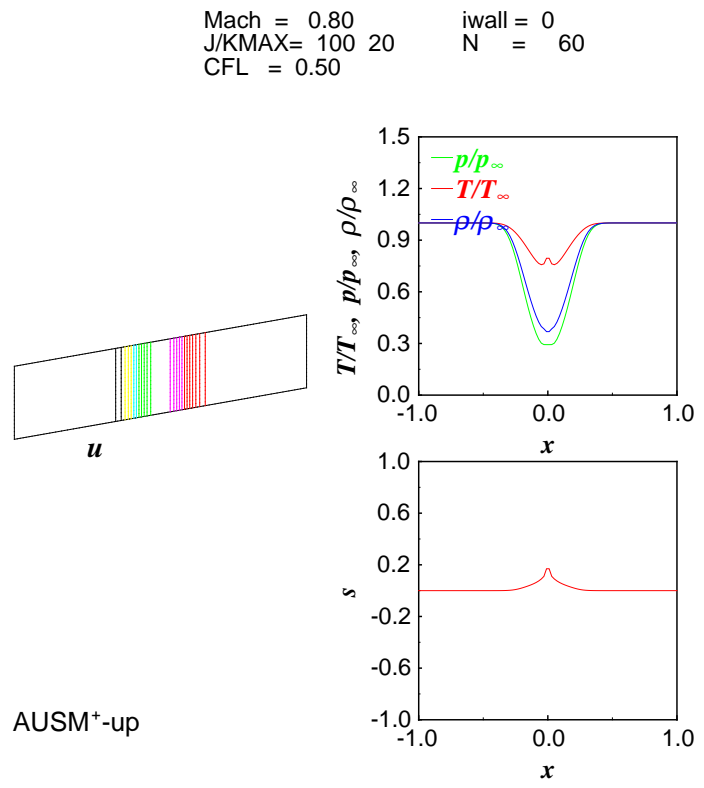

(a) $\mathrm{AUSM}^{+}$-up

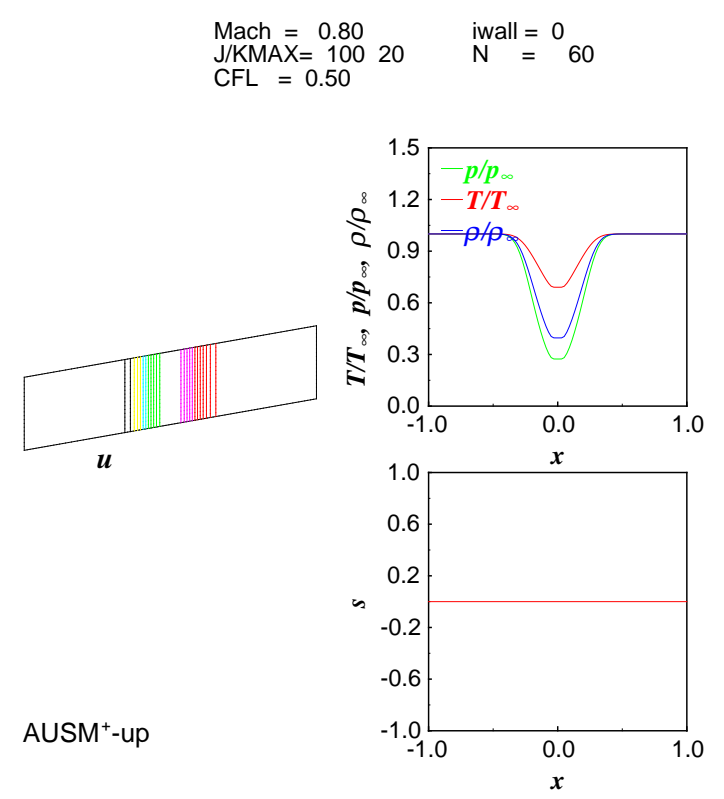

(b) $\mathrm{AUSM}^{+}$-up with s-equation

Figure 8. Effects of enforcing the entropy equation. (a) $\mathrm{AUSM}^{+}$-up and (b) $\mathrm{AUSM}^{+}$-up with the entropy equation for a $2 \mathrm{D}$ receding flow problem (M=0.8).

\footnotetext{
${ }^{\mathrm{b}}$ The conservation form is not unique as it can in general appear as $\partial \rho s^{m} / \partial t+\partial \rho u s^{m} / \partial x=0, m=1,2, \ldots$
} 
In what follows we shall consider some relevant 2D rarefaction problems. The $2 \mathrm{D}$ version of the entropy equation is applied. First we calculate a $2 \mathrm{D}$ receding flow, defined by the initial condition:

$$
u_{R}=-u_{L} \neq 0, \quad v_{R}=-v_{L}=0, \quad p_{R}=p_{L}, \rho_{R}=\rho_{L},
$$

where $u$ and $v$ are the $x$ and $y$ components of the velocity, respectively. The flow is calculated on a 2D slanted mesh. The results obtained by using the energy equation and the entropy equation are given in Fig. 8. As in previous examples, overheating is seen with the conventional formulation, while the entropy equation gives rise to the expected correct result in which the entropy remains constant and the temperature behaves properly.

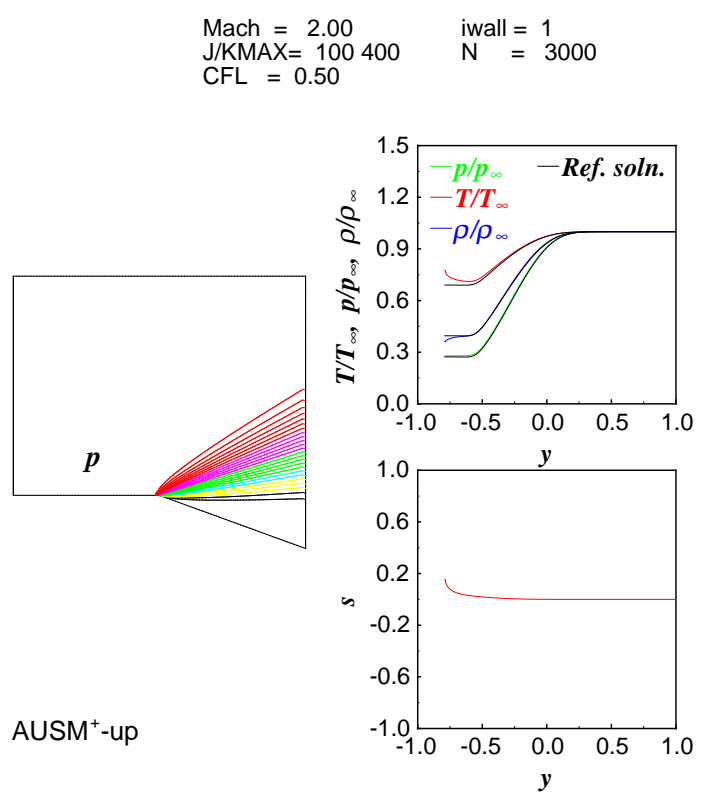

(a) $\mathrm{AUSM}^{+}$-up

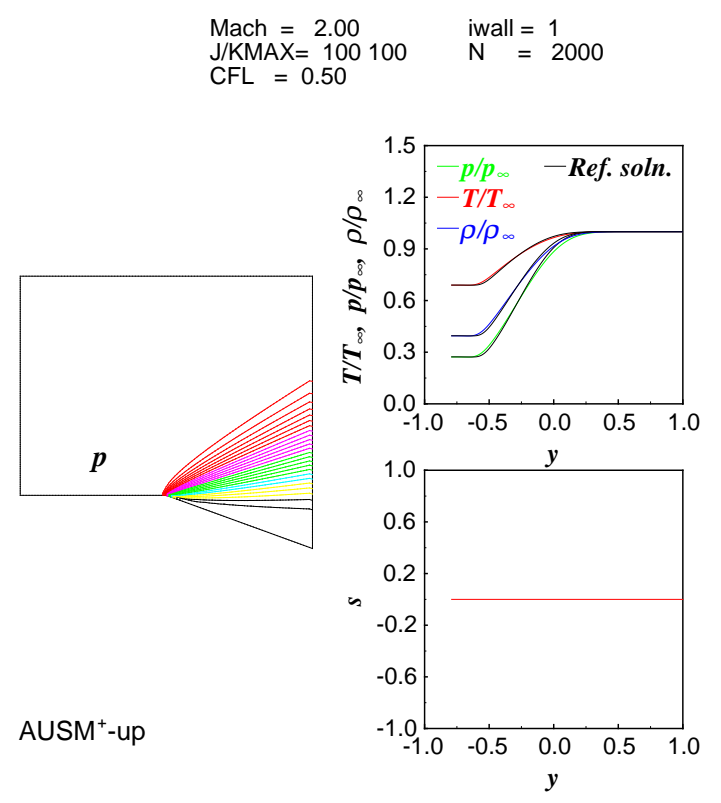

(b) $\mathrm{AUSM}^{+}$-up with s-equation

Figure 9. 2D Prandtl-Meyer flow $\left(\mathbf{M}=2.0\right.$, corner angle of $20^{\circ}$. (a) $\mathbf{A U S M}^{+}$-up $\left(100 \times 400\right.$ mesh) and (b) $\mathbf{A U S M}^{+}$-up with the entropy equation $(100 \times 100$ mesh); reference solution is obtained with a $100 \times 400$ mesh.

Next we consider a supersonic $(M=2.0)$ flow over an expansion corner of $20^{\circ}$, or the Prandtl-Meyer flow. Here we show the calculated results without and with using the entropy equation; the inaccurate prediction of the wall temperature, namely overheating, is clearly seen in Fig. 9 when the conventional conservation equations are employed, even with a very fine mesh of $100 \times 400$. On the other hand, the entropy equation gives a correct wall temperature and excellent agreement with the reference solution, with a coarse mesh of $100 \times 100$. The reference solution refers to the isentropic solution on the mesh of $100 \times 400$.

Table 2 gives the comparison of calculated wall temperature with different grid sizes; it shows that the result is hardly improved by reducing the grid spacing by eight times when the entropy condition is violated; the error from the reference solution remains about $10 \%$. Interestingly, the isentropic solution on the coarsest mesh (100) already gives a wall temperature essentially identical to the reference value.

Table 2. Comparison of predicted wall temperature with different grid sizes to the reference value.

\begin{tabular}{|c|c|c|c|c|c|c|} 
Grid points & $N_{y}=50$ & $N_{y}=100$ & $N_{y}=200$ & $N_{y}=400$ & s-eqn, $N_{y}=50$ & Reference solution \\
\hline$T_{\text {wall }}$ & 0.7793 & 0.7767 & 0.7754 & 0.7742 & 0.6893 & 0.6900 \\
\hline
\end{tabular}

It must be reminded that while the use of the entropy equation cures the overheating problems, its applicability is limited to smooth flows only. Even within the realm of inviscid flow, many are nonsmooth and involve shocks. Including the entropy equation in the system will still yield discontinuous solutions across a shock but with entropy unchanged, hence its strength is incorrect. For transonic flows, the error is small, $\left(O(M-1)^{3}\right)$, this formulation nevertheless is not general. We use the entropy equation only to provide the numerical evidence for the analysis developed above that the entropy generation is the reason for the temperature anomaly. 


\section{Conclusion}

We have achieved the objective of this study: to uncover the root cause of the overheating in the smooth receding problem. The root cause of the overheating has its origin in the entropy generation $a b$ initio, which is tied to the pressure flux in the finite volume discretization of the conservation laws. The overheating is found to be inevitable for all numerical flux schemes used, as confirmed in the numerical tests. The rate of overheating is proportional to the flow separation speed and is a function of flux scheme.

To support the conclusion of the analysis, an isentropic system is used: the entropy equation is employed to replace the energy equation. The use of the entropy equation proves that incorporating the entropy condition in the discretized system eradicates the temperature overheating problem, ensuring the correct temperature and entropy distributions. Its efficacy has been demonstrated in several relevant rarefaction problems. Especially the inaccuracy in the calculated wall temperature for the 2D Prandtl-Meyer problem by the conventional finite volume approach may have a practical implication.

Finally, we also remind that the applicability of the entropy equation is limited to isentropic flows which in practice are rather rare. Its usage in this study is only to provide numerical evidence of the root cause of the overheating problem and is not recommended for general problems. However, if an occasion of inaccurate temperature prediction arises for an isentropic flow, we now know the cause and how to fix it.

\section{Acknowledgment}

The author gratefully acknowledges the support of the Transformational Tools and Technologies and Advanced Air Transport Technologies Projects for this research, Jeffery Moder and Willian Haller are the technical lead respectively.

\section{References}

\footnotetext{
${ }^{1}$ Liou, M.-S., "Open problems in numerical fluxes: proposed resolutions," AIAA Paper 2011-3055, 2011.

${ }^{2}$ Toro, E. F., Riemann solvers and numerical methods for fluid dynamics: a practical introduction, Springer-Verlag, Berlin, Heidelberg, 3rd ed., 2009.

${ }^{3}$ LeVeque, R. J., Numerical Methods for Conservation Laws, Birkhauser Verlag, Basel, 1990.

${ }^{4}$ Liou, M.-S., "Unresolved problems by shock capturing: Taming the overheating problem," 7th International Conf. on Comput. Fluid Dyn. 2012-2203, 2012.

${ }^{5}$ Liou, M.-S., "Why is the overheating problem difficult: The role of entropy," AIAA Paper 2013-2697, 2013.

${ }^{6}$ Noh, W. H., "Errors for calculations of strong shocks using an artificial viscosity and an artificial heat flux," J. Comput. Phys., Vol. 72, 1978, pp. $78-120$.
} 